\title{
Artificial Neural Networks for the prediction of the trapping efficiency of a new sewer overflow screening device
}

\author{
$\underline{\text { M.A. Aziz }}^{\text {a }}$, M.A. Imteaz ${ }^{\text {a }}$, T.A. Choudhury ${ }^{\text {a }}$ and D.I. Phillips ${ }^{\text {b }}$ \\ ${ }^{a}$ Faculty of Engineering and Industrial Science, Swinburne University of Technology \\ Melbourne, Victoria 3122, Australia. ${ }^{b}$ Water Solutions (Aust) Pty Ltd \\ Melbourne, Victoria 3926, Australia \\ Email: mimteaz@swin.edu.au
}

\begin{abstract}
Some of the major concerns regarding sewer overflows to receiving water bodies include serious environmental, aesthetic and public health problems. Water management authorities are increasingly receiving public complaints that have led engineers to focus on means of retaining the entrained sewer solids within the sewer system during overflow events. During wet weather conditions, sewer overflows to receiving water bodies raise serious concern to environmental and community health concerns. To address these problems, different types of screening devices are used. Moreover, floatable control is preferred by most of the proposed and existing environmental regulations. This requirement triggers the need to research the different types of screening devices and screenings handling systems to select the most appropriate for a particular installation especially at unmanned locations.
\end{abstract}

In the present study the sewer overflow device consists of a rectangular tank and a sharp crested weir that are followed by series of vertical parallel combs to separate entrained sewer solids from the overflow. The device does not require electrical or mechanical power for the self-cleansing mechanism, enabling the device to work efficiently in unmanned locations. Extensive laboratory investigations are underway to assess the effectiveness of a novel self-cleansing sewer overflow screening device. A series of laboratory tests to determine trapping efficiencies for common sewer solids were conducted for different flow conditions, number of combs layers, spacing of combs and weir crest lengths.

Sewer solids from different density materials make sewer flow to analyze in complex Non-Newtonian fluid system with huge computational cost and complicity using physical law based modeling. On the flipside artificial neural model has the capacity to accurately predict the outcome of complex, non-linear physical systems with relatively poorly understood physicochemical processes which makes them highly desirable in the present study. Artificial Neural Networks (ANN) have already been successfully used to simulate flood forecasting in urban drainage system, real time control in combined sewer system, real time water level predictions of sewerage systems covering gauged and un-gauged sites etc.

In case of sewer solid capture efficiency: neural network modeling is able to recognize nonlinear input output relations with adapting approach for changing circumstances. In the present study, feed forward artificial neural networks using back propagation algorithms were used, as such networks have been used almost exclusively in environmental modeling. A series of forty seven (47) sets of experimental data were collected to train (calibrate) the ANN model. In addition to these, eight (8) sets of experimental data were collected to validate the trained ANN network to be used in wider prospective of urban drainage conditions.

The major areas covered in the ANN modeling include selection of input and output variables, optimization of the model, consideration of different learning algorithms, designing ANN's training \& cross training processes and model validation. In the studied case, complex physical characteristics of different sewer solids, together with multi-fluid sewer system with variable flow phenomena makes it difficult to model with physical considerations. In case of sewer solid capture efficiency; artificial neural network modeling is able to learn the complex input-output relations with adapting approach for changing circumstances. Model considered different learning algorithms, diverse hidden layer structure with varied training samples to optimize the network. It is found that the model can successfully predict the experimental results with average absolute percentage errors varying from 4 to 7 percent.

Keywords: Screening device, sewer solids, capture efficiency, and artificial neural network 
Aziz et al., Artificial Neural Networks for the prediction of the trapping efficiency...

\section{INTRODUCTION}

Under wet weather conditions, sewer overflows cause serious concern to the environment, aesthetic and public health when present in receiving water bodies. To overcome these problems different types of screening devices are used. Screening is a process that is most desirably confined and automated in order to ensure operational safety and to satisfy aesthetic issues. Moreover, floatable control is preferred by most proposed and existing environment regulations. This requirement triggers the need for research in the construction of numerous screening handling systems in actual environments, especially at unmanned locations where minimum maintenance is available. With the advent of powerful computing facilities over the last couple of decades, computer models have been extremely useful in devising and assessing the various engineering and operational strategies for managing water quality and quantity. In the case studied, ANN models were suitable considering mixing of different solids in the sewer system which leads to different viscosity fluid flow conditions. Moreover, the nonlinear relationship between input and output variables, and complex physicochemical interaction leads to difficulties in formulating mathematical model based on physical laws.

There are a number of different screening systems used in sewer overflow screening devices. One of these is the rotary screen (Moffa, 1997), consisting of a large rotating drum; slightly angled to maximize dewatering. The angle of the drum ensures effective dewatering once the screenings travel up the drum before reaching the end of the drum, at which point the screenings are removed from the unit. Metcalf et al. (1991) proposed a centrifugal screen, having a series of screens attached to a cage, which rotates around a vertical axis. Flow enters the inside from the bottom and flows upward to a deflection plate at the top of the unit which can be collected from outside the cage. Other types include disc type plates, oriented perpendicular to the flow and surrounded with screening media, that rotate about a horizontal axis. The solids are retained by the screening media after the influent enters the submerged portion of the disc. A cleansing brush mechanism known as the brush raked fine screen is mounted on a drive shaft that slowly rotates in a 360 degree circle. In addition to these, Moffa(1997) used an inclined static screen that acts as a sieve to remove solids from the liquid stream. An overflow sewer device weir screen acts as a barrier to retain floatable and other solids and the rotating weir screen is cleaned by a rotating brush that is powered by the energy of water flowing over a water wheel or by an electric motor. Simon et al. (2008) proposed a sewer overflow screening device with temporary holding tanks, which provide transient storage and real time control of sewer systems. Although the device was self cleansing, the cleansing mechanism had some limitations which needed to be improved.

Some of the common drawbacks in the available commercial devices include inadequate screening capacity, external power needs and high cost. To overcome such drawbacks a new overflow sewer device, known as the Comb Separator, was proposed by Donald Phillips (Phillips et al., 2010). The device has no moving parts, a robust stop/start operation, an effective self cleansing mechanism, low maintenance and operation costs and no external power requirements. World-wide patents for the device have been applied for and licenses issued for its manufacture and marketing.

In the present study, the sewer overflow device consists of a rectangular tank and a sharp crested weir that are followed by series of vertical parallel combs to separate entrained sewer solids from the overflow. The device does not require electrical or mechanical power for the self-cleansing mechanism, enabling the device to work efficiently in unmanned locations. In addition, it has no moving parts and has robust stop/start operation and low maintenance. The performance of the device is based on its sewer solids capture efficiency. A series of laboratory tests conducted at Swinburne University of Technology used a range of experimental conditions to simulate conditions in existing urban sewerage systems. These included different overflow, or spill rates, for different numbers of combs layers, spacing of combs and weir crest lengths. The experimental work although showing promise as a sewer solids separation device, was restricted by the physical limitations inherent in laboratory studies. In addition, experimental work involves significant cost and time. To overcome such problems experimental results were analyzed and used to train an ANN model that had already been used successfully in similar kinds of environmental problems like water level predictions, flood forecasting and control in combined sewers (Chiang et al., 2010, Bruen et al., 2006 and Weyand, 2002).

Willems et al. (1999) demonstrated a number of uncertainties involved in the modeling of a sewer system. In particular, model simplifications of the physical system make it difficult to adopt a deterministic approach. In the studied problem, the system difficult to model: (i) physical characteristics of different sewer particles; (ii) multi-fluid sewer system with changing velocity due to different viscosity of fluid (as Non-Newtonian fluid); and (iii) interaction between liquid and solid particles. On the other hand, the main benefit of adapting ANNs are that they can effectively extract significant features and trends from complex systems even if the underlying physics is either unknown or difficult to recognize (Chiang et al., 2010). Furthermore, ANNs 
greatly reduce the computational time and cost (Raduly et al., 2007). In addition to these, once the network is trained it reduces computation cost and time unless completely new sets of experimental conditions are used which could be greatly enhancing industrial applicability. Saving in computational cost, time and predict complex input output relations without much understanding of physicochemical system could make an artificial neural network (ANN) model the obvious choice in a wide range of urban drainage systems.

In the case of sewer solid capture efficiency, the neural network modeling is able to learn the existing nonlinear input- output relationships. In this work, a multi-layer feed forward artificial neural network, using back propagation algorithm, were used. Such networks have been used almost exclusively in environmental modeling (Maier et al., 2001). A series of forty seven (47) sets of experimental data were collected to train the ANN model. In addition to these, eight (8) sets of experimental data were collected to validate the trained ANN models. They are to be used in wider perspective of urban drainage conditions.

\section{SCREENING MECHANISM}

The proposed overflow sewer device is installed within existing sewer overflow chambers. The sewerage overflow device is connected to a pump and inlet pipe, two outlets are mounted on the device one to convey away overflow water whereas the other drains the sewer water remaining in the storage chamber. A series of combs to segregate sewer solids from the sewer overflow are mounted next to the sharp crested weir as shown in figure 1 below.

After the start of precipitation the storage chamber fills with sewerage. A floating valve at the bottom of the sewer solids holding chamber then closes at this point as shown in figure 1 . As the downpour continues the storage chamber overflows the sharp crested weir and the entrained sewer solids are intercepted by the parallel combs and fall into the holding chamber (pollutant capture chamber) refer to figure 1. After cessation of the down pour the storage chamber falls below the valve level.

The pressure of the water in the sewer solids holding chamber opens the valve and flushes the entire captured sewer solids back into the storage chamber refer to figure 2. Common sewer solids like condoms, tampons, cigarette buds, wrap papers, bottle caps were tested. The experimental conditions varied with different flow conditions, number and spacing of combs layers. The laboratory device gave very high capture rates varying from $90 \%$ to $95 \%$ percent with the exception of cigarette butts.

\section{ARTIFICIAL NEURAL NETWORK MODELING}

The Artificial Neural Network (ANN) is highly inspired by biological neural networks and has the unique ability to learn and generalize "knowledge". ANNs are a non-linear data modeling tool used to model complex relationships between inputs and outputs without any prior assumptions or any available mathematical relation between them. ANNs comprise a group of interconnected artificial neurons, which are the simple and fundamental processing units. Each artificial neuron (Figure 3 ) is basically a computing processor, where the output $y_{k}$ is a function of the weighed sum of the inputs eqn. (1). Where, $x_{1}, x_{2}, \ldots, x_{p}$ are the input signals; $w_{k l}, w_{k 2}, \ldots, w_{k p}$ are the assigned weights; $\theta_{k}$ is the threshold value and $\varphi$ is the transfer function .

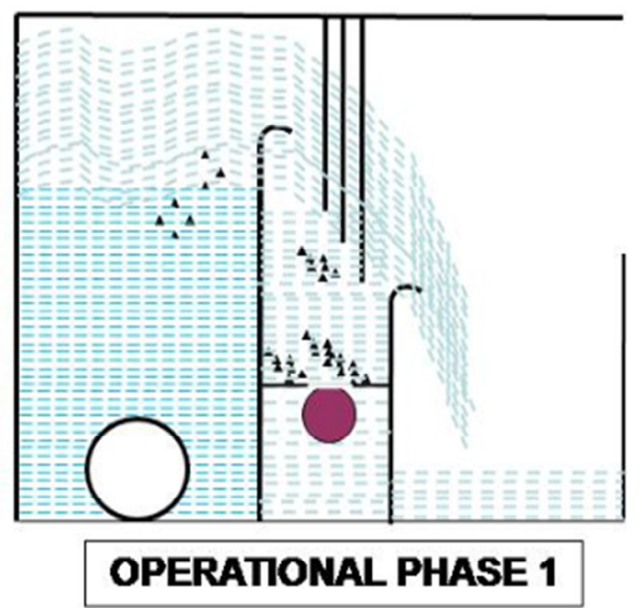

Figure 1.Operation procedure of the new Overflow Sewerage Device.

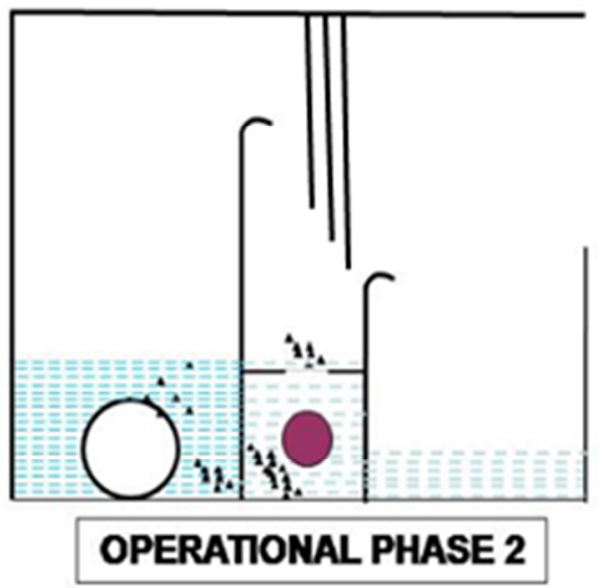

Figure 2.Operation procedure of the new Overflow Sewerage Device. 


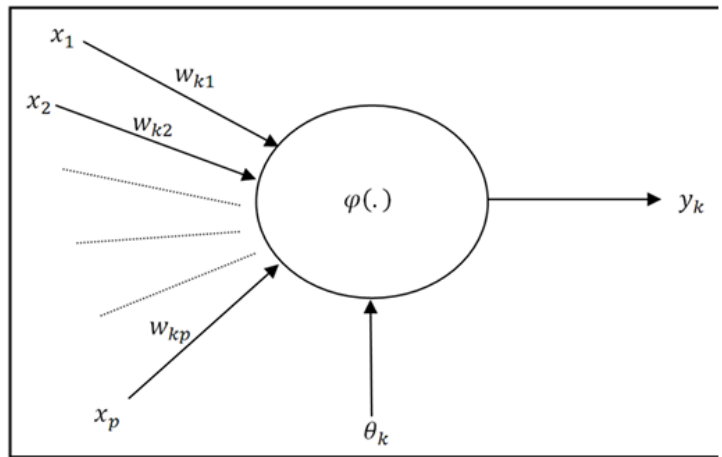

Figure 3.A typical artificial neuron $k$. $y_{k}=\varphi\left(\sum_{j=1}^{p} w_{k j} x_{j}-\theta_{k}\right)$

Artificial Neural Networks have already been successfully used to simulate flood forecasting in urban drainage system (Bruen et al,2006), real time control in combined sewer systems in Germany (Weyand et al,2002), real time water level predictions of sewerage systems covering gauged and ungauged sites (Chiang et al, 2010). As ANNs have been successfully applied to similar nature of water quality applications (Maier et al, 1996) it is also used in the current study.

The major areas covered in ANN modeling include selection of input and output variables, optimization of the model, consideration of different learning algorithms in building and training networks and finally model validation (Maier et al, 2001).

\subsection{Selection of input-output variables}

Like most environmental modeling approaches, some of the key steps in ANN modeling include database collection (from experimental results), pre-possessing the data (normalize experimental data) and assessing the output. A robust and sufficiently large database is essential to construct a network that generalizes well. Moreover, a clear understanding of the hydraulic process is required for successful modeling of this nature. For instance, physical insight into the problem being studied can lead to better choices of input variables for proper mapping (ASCE, 2000). This will lead to effective and efficient modeling, avoiding loss of information due to inappropriate choice of input parameters. In the present study 47 sets of experimental data showed that 16 input parameters influenced the pollutant capture efficiency (output). While carrying out the experiment, one parameter was varied each time, while the others were kept constant to their reference values. This allows us to examine how each parameter affected the output pollutant capture efficiency.

\subsection{Model Design and Network Optimization}

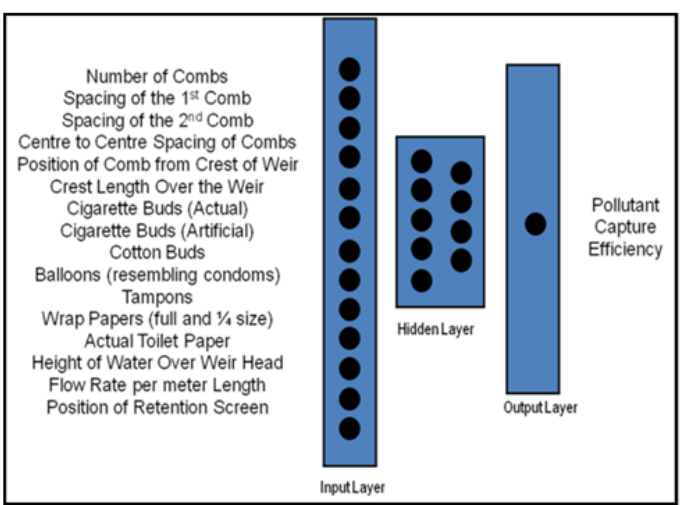

Figure 4.Block diagram of proposed ANN model
A model considering Multi-Layer Perceptron (MLP), based on the back propagation algorithm, is used in this work. The multi-layer ANN architecture comprises three main parts: the input layer, the output layer and the layer in between termed as the hidden layer. The input parameters were selected based on the experience on extensive hydraulic experimental works on the sewer solid capture efficiency. Therefore 16 parameters affecting the pollutant capture efficiency were considered for inputs, where as pollutant capture efficiency was selected as output parameter. The number of neurons required to describe each parameter is dependent on the parameter nature. A real valued parameter requires one neuron to represent the value, while $\mathrm{x}$ neurons are required to describe $2^{\mathrm{x}}$ categories for parameters representing classifications. The flexibility lies in selecting the number of hidden layers and in assigning the number of neurons to each of these layers. Maren et al (1990) suggests using two hidden layers when the outputs need to be continuous functions of the inputs. The block diagram of the proposed ANN model is presented in Figure 4.

An optimal architecture may be considered the one yielding the best performance in terms of output error minimization, while retaining a simple and compact structure. The network optimization uses the experimental database to fix the number of neurons in the hidden layers as well as optimize the weight population to produce minimum output error. 
The training process for ANNs can be considered similar to the idea of calibration that is an integral part of most hydrologic modeling studies. The purpose of training is to determine the set of connection weights and nodal thresholds that cause the ANN to predict outputs that are sufficiently close to target values (ASCE, 2000). To avoid the problem of over-fitting and to improve the generalization ability of the trained network, the method of cross-validation and early stopping are implemented. The available dataset is divided into three parts: the training set, the validation set and the test set. In present study, we used $60 \%$ of the data for training set and $20 \%$ for each of the validation and test sets. The back propagation algorithms initially selected in this study are the Levenberg Marquardt and the Resilient Back Propagation.

The Levenberg-Marquardt algorithm (Hasan et al, 1994) is an approximation to Newton's method designed to reach the second order training speed without computing the Hessian matrix. The approximation of Hessian matrix and the error gradient is computed as per Eqs (2) and (3).

$$
H=J^{T} J \quad(2) \quad g=J^{T} e
$$

$J$ represents the Jacobian matrix, generated by the first derivatives of the network errors on the training set with respect to the network's weights and biases. This can be calculated using standard back propagation technique (Hasan et al, 1994). In Eq. 3 e represents the network errors.

The Levenberg Marquardt algorithm uses the approximation in calculating the Hessian matrix to update and tune the parameters. If $z_{k}$ represents the old parameter value, then the new parameter value is given by Eq. 4 .

$z_{k+1}=z_{k}-\left[J^{T} J+\mu I\right]^{-1} J^{T} e$

The Resilient Back Propagation algorithm (Riedmiller et al, 1993) eliminates the harmful effects of the magnitudes of the partial derivatives as we use sigmoid transfer functions in compressing an infinite input range into a finite output range. These sigmoid functions are characterized by their slopes approaching zero as the input gets large. This creates difficulty as the gradient can have a very small magnitude and, therefore, cause small changes in the weights and biases, even though the weights and biases are far from their optimal values. In resilient back propagation algorithm, only the sign of the derivative can determine the direction of weight update.

The number of neurons in the first and second hidden layer was varied from combination of 5/4 neurons to 23/22 neurons. Regression analysis was performed between the predicted and experimental pollutant capture efficiency values. From the performed regression analysis, it was found that the ANN structure with 5/4 neurons in the first and second hidden layer respectively, responds well both to Levenberg Marquardt and Resilient Back propagation algorithm with high R-value. The regression analysis performed on the training,

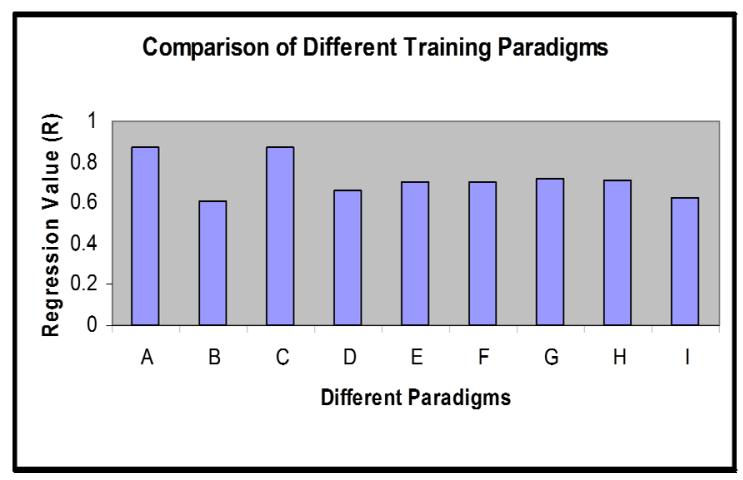

Figure 8. Comparison of different training paradigms

Where alphabets A, B, C ...in figure 8 represents: A: Levenberg-Marquad; B: BFGS Quasi-Newton; C: Resilient Backpropagation; D: Scaled Conjugate Gradieent; E: Conjugate Gradient with Power/ Beale Restarts; F: Fletcher-Powell Conjugate Gradient; G: Polak-Ribiere Conjugate Gradient; H: One Step Secant; I: Variable Learning Rate Back Propagation. validation and test sets for the ANN with five and four neurons in the first and second hidden layer respectively and trained with Resilient Back propagation algorithm is shown in Figure 6 and 7 respectively.

To ensure that the Levenberg Marquardt algorithm and the Resilient Backpropagation algorithm are the optimal training algorithm for the current problem of modeling capture sewer overflow efficiency, the network with five and four neurons in the first and second hidden layer respectively, is trained and simulated with nine different algorithms, including the ones the network was trained with previously. Figure 8 summarizes the regression analysis performed on each of the trained networks. 30 different network weight trials were given in each steps where different random initial weights are used in each trial and best values for regression analysis (R) were collected. Both Levenberg-Marquardt and Resilient Back propagation algorithms revealed the highest regression value $(R)$ value of $R=0.862$. 


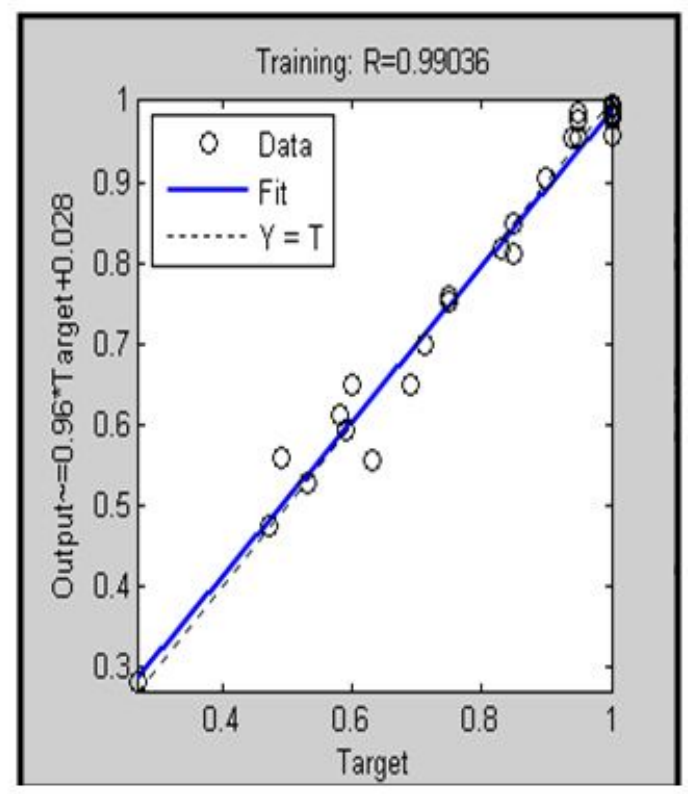

Figure 6.Training data for ANN model

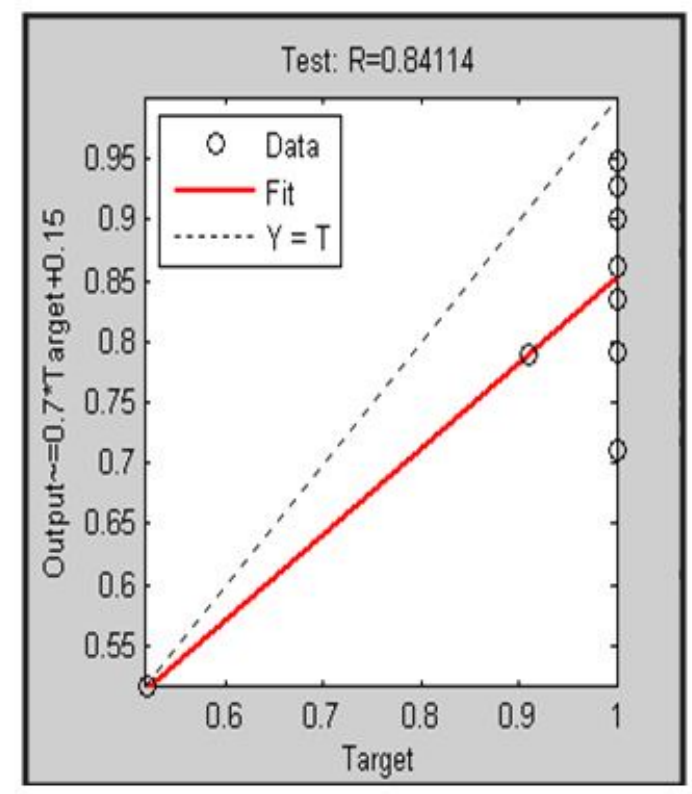

Figure 7.Training data for ANN model

\subsection{ANN model validation}

Once an ANN model has been trained successfully, the model validation needs to fairly evaluate it by subjecting it to new patterns that it has not seen during training. A new test set is used for this purpose. In the

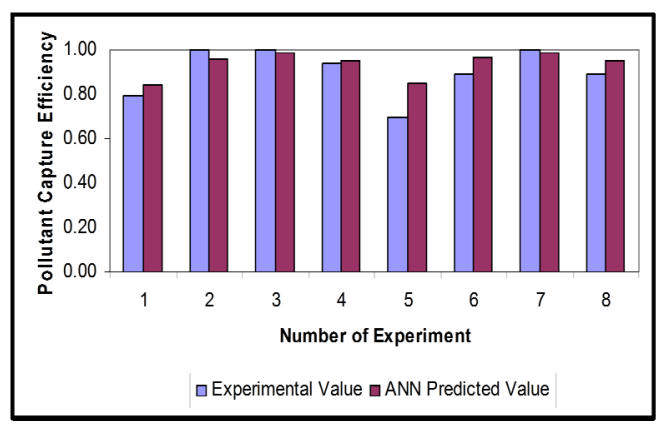

Figure 9.Comparison of experimental values and ANN predicted values. studied problem eight new sets of experimental data were collected and they were simulated with the trained network to obtain the predicted values. The generalization performance of a trained network is measured on the error it produces on unknown data. The smaller is the error, the better the generalization ability of the trained network, which means that the network will perform better under unknown and unseen environments. It was found that the trained ANN successfully predicted the new experimental values with error ranges from $4 \%$ to $7 \%$ (Figure 9), which shows that the network was trained properly with good generalization ability.

\section{DISCUSSION OF RESULTS}

Our limited understanding of physics with NonNewtonian fluids made it quite complicated and time consuming to model the system using a deterministic approach. On the flip side of the coin, the empirical knowledge through a series of experiments leads us to formulate various assumptions by which to successfully develop the ANN model. The ANN's powerful modeling approach, when trained with input-output data, shows that the model can mimic the underlying hydrologic processes that otherwise would be extremely difficult to model. It can also handle noise in the input output data quite efficiently without severe loss of accuracy (ASCE, 2000). Moreover the ANN model greatly reduces computational time compared with most mathematical models (Radulyet al, 2007). All these attributes, along with the nonlinear nature of the activation function, truly enhance the generalization capabilities of ANNs in the studied problem. Special attention was given to the generalization of errors during test cases with different algorithms which significantly contributed to the ANNs performance in predicting experimental pollutant capture efficiency as shown in figure 10.After a significant trial and error process, the optimized ANN model was designed and successfully predicted experimental results with mean absolute error of around 5\% (shown in figure 9). This demonstrates the ability of the model

To predict sewer solid capture efficiencies of the device under real-world conditions. The results presented in this paper confirm that ANNs have high potential in predicting pollutant capture efficiencies of the sewer overflow device. For integrated urban waste water system simulations, where repeated training of the ANN is not needed, ANN greatly reduces simulation time when compared with deterministic modeling approaches. 
Aziz et al., Artificial Neural Networks for the prediction of the trapping efficiency...

\section{CONCLUSION}

From experimental data the operational efficiency of a new sewer overflow screening device was proven that ensures effective screening and has a self cleansing mechanism and a robust start/stop operation. In order to employ it in urban drainage systems, neural network modeling was proposed. The model overcomes some common experimental drawbacks such as different scales, structures and the time and cost involved in the experimental processes. In order to optimize performance of the model the following issues were given consideration.

- Determination of appropriate model inputs: Due to availability of the experimental apparatus, the better understanding of the physical aspects lead to a better choice of the sixteen input variables for proper mapping on the output.

- The choice of adequate network geometry: A model considering multi-layer perception (MLP) on a Resilient Back-propagation algorithm having 5 neurons in the $1^{\text {st }}$ hidden layer and 4 neurons in the $2^{\text {nd }}$ hidden layer was found to be the optimum for the test device.

- Network behavior during the parameter estimation phase: Sixty percent of the data was used in training where as twenty percent of the data was used for the validation and testing phases.

- Model validation: The ANN model successfully predicted the experimental results with errors varying from 4 to 7 percent.

The model can be used as an integrated approach with the device in the urban sewer system which will effectively work to remove sewer solids from overflows from the existing sewer system thus helping to improve the water quality and ecology of receiving waters.

\section{REFERENCES}

ASCE Task Committee on Application of Artificial Neural Networks in Hydrology (2000). Artificial neural networks in hydrology I \& II. Journal of Hydrologic Engineering, , 5(2),April.

Bruen, M., Yang, J. (2006). Combined hydraulic and black-box models for flood forecasting in urban drainage systems, Journal of Hydrologic Engineering, 11(6), November.

Chiang, Y.M., Li-Chiu Chang, Tsai, M.J.,Wang, Y.F., and Chang, F.J.(2010). Dynamic neural networks for real-time water level predictions of sewerage systems-covering gauged and ungauged sites. Journal of Hydrology and Earth System Sciences, 14, 1309-1319.

Maier, H. R., and Dandy, G.C. (2001). Neural network based modelling of environmental variables: asystematic approach, Journal of Mathematical and Computer Modelling, 33, 669-682.

Maier, H. R., and Dandy, G.C. (1996). The use of artificial neural networks for the prediction of water quality parameters, Journal of Water Resources Research, 32(4), 1013-1022, April.

Metcalf and Eddy (1991). Wastewater engineering, treatment, disposal and reuse, Third Ed., Boston, MA: McGraw-Hill, Inc. 448-451.

Maren, A., C. Harston, and R. Pap (1990). Handbook of neural computing applications, academic, San Diego, Calif, 448.

Moffa, P.E., (1997). Scarborough CSO project-review of CSO screen application, report prepared for the City of Scarborough, Municipality of Metro, Toronto, ON.

Hagan M.T., Mehnaj, M.B.(1994). Training Feedforward Networks with the Marquardt Algorithm, IEEE

Transactions on Neural Networks, 5 , 989-993.

M. Riedmiller,M. . Braun,H.,(1993) in: Anon (Ed.), Direct adaptive method for faster backpropagation learning: The RPROP algorithm, Publ by IEEE, San Francisco, CA, USA, pp. 586-591.

Phillips, D.I. and Simon, M. (2010). An improved method of screening sewer solids during CSO events. Proc. of the 7th Int. Novatech Conf. On Urban Drainage, Lyon, France.

Raduly, B., Genaey, K.V., Capodaglio, A.G., Mikkelsen, P.S., Henze, M. (2007). Artificial neural networks for rapid WWTP performance evaluation: methodology and case study, Journal of Environmental Modelling and Software, 22,1208-1216.

Simon. M. and Phillips. D.I. (2008). The development of a sewer solids screening system for CSO chambers. Proc. of the 11th Int. Conf. on Urban Drainage, Edinburgh, Scotland, UK.

Weyand,M. (2002). Real-time control in combined sewer systems in Germany-some case studies, Journal of Urban Water, 4, 347-354.Willems, P., \& Berlamont, J. (1999). Probabilistic modelling of sewer system overflow emissions. Water Sci. Tech, 39(9),47-54. 